Coopérative et rapport au travail : le cas d'une CAE The relationship to work in a cooperative: The case of the business and employment cooperative (CAE)

Cooperativa y relación con el trabajo: el caso de una cooperativa de actividades y empleo (CAE)

\title{
Marc-André Vilette
}

Numéro 338, octobre 2015

ESS et mutations du travail et de l'emploi

URI : https://id.erudit.org/iderudit/1033874ar

DOI : https://doi.org/10.7202/1033874ar

Aller au sommaire du numéro

\section{Éditeur(s)}

Association Recma

\section{ISSN}

1626-1682 (imprimé)

2261-2599 (numérique)

Découvrir la revue

\section{Citer cet article}

Vilette, M.-A. (2015). Coopérative et rapport au travail : le cas d'une CAE. Revue internationale de l'économie sociale, (338), 69-84.

https://doi.org/10.7202/1033874ar

\section{Résumé de l'article}

La coopérative d'activités et d'emploi (CAE) permet d'accompagner les porteurs de projets entrepreneuriaux. A ce titre, les particularités de la CAE devraient avoir une influence sur eux - sur leurs compétences et leur identité professionnelle en particulier -, sous le statut original d'entrepreneurs-salariés, et plus généralement modifier leur rapport au travail. Cet article mobilise, dans un premier temps, la littérature sur la CAE, les profils génériques d'entrepreneurs, leurs compétences et leur identité professionnelle. Dans un second temps est présentée une enquête exploratoire dans une de ces coopératives, qui infirme certains aspects de la revue de littérature. 


\title{
COOPÉRATIVE ET RAPPORT AU TRAVAIL: LE CAS D'UNE CAE
}

\author{
par Marc-André Vilette*
}

\begin{abstract}
* Ecole supérieure de commerce (ESC) de Saint-Etienne et Ecole supérieure de commerce (ESC) de Clermont.
\end{abstract}

La coopérative d'activités et d'emploi (CAE) permet d'accompagner les porteurs de projets entrepreneuriaux. A ce titre, les particularités de la CAE devraient avoir une influence sur eux - sur leurs compétences et leur identité professionnelle en particulier -, sous le statut original d'entrepreneurs-salariés, et plus généralement modifier leur rapport au travail. Cet article mobilise, dans un premier temps, la littérature sur la CAE, les profils génériques d'entrepreneurs, leurs compétences et leur identité professionnelle. Dans un second temps est présentée une enquête exploratoire dans une de ces coopératives, qui infirme certains aspects de la revue de littérature.

The relationship to work in a cooperative: The case of the business and employment cooperative (CAE)

The business and employment cooperative (coopérative d'activités et d'emploi or CAE) provides support for the self-employed starting their own businesses. As such, the particular characteristics of the CAE should have an effect on these self-starters - on their competences and their professional identity in particular - in their unique capacity as an entrepreneur-employee and, more generally, alter their relationship to work. In the first part, this article introduces the literature on the CAE, the generic profiles of entrepreneurs, their competences and their professional identity. In the second part, an exploratory survey in one of these cooperatives is presented, which contradicts certain aspects in the review of the literature.

\section{Cooperativa y relación con el trabajo : el caso de una cooperativa de actividades y empleo (CAE)}

La cooperativa de actividades y empleo (CAE) permite acompañar los responsables de proyectos empresariales. En este sentido, las particularidades de la CAE deberían influir estos responsables - particularmente sus competencias y su identidad profesional -, con el estatus original de empresario-asalariado, y mas generalmente cambiar su relación con el trabajo. Este articulo moviliza, en primer lugar, los estudios sobre la CAE, los perfiles genéricos de empresarios, sus competencias y su identidad profesional. En segundo lugar, se presenta una investigación exploratoria en una de estas cooperativas, que desmiente algunos aspectos de los estudios. 
L'adoption par le Parlement le 31 juillet 2014 d'une loi spécifique à l'économie sociale et solidaire (ESS) illustre l'importance croissante de celle-ci. Au cours de ces dernières décennies en effet, ni l'économie capitaliste, ni l'économie publique n'ont pu répondre à une certaine demande sociale, favorisant ainsi le développement de l'ESS (Gianfaldoni, Richez-Battesti, 2005).

Afin de répondre à cette demande sociale (Charles Pauvers, Schieb-Bienfait, Urbain, 2011), l'entrepreneuriat apporte en particulier des solutions originales à l'insertion pour les demandeurs d'emploi. Il peut même permettre aux plus défavorisés de sortir de l'exclusion (Brasseur, 2009).

La coopérative d'activités et d'emploi (CAE) représente une de ces formes d'entreprise sociale (Draperi, 2010). Cependant, ce type de structure relativement nouveau est encore peu développé, ce qui explique sans doute une littérature assez récente. Au regard de celle-ci, nous avons donc souhaité examiner plus particulièrement certaines caractéristiques des porteurs de projet entrepreneurial accueillis en CAE peu étudiées, notamment leurs compétences individuelles et leur identité professionnelle. Ce choix se justifie d'autant plus que «la CAE permet de se réapproprier l'acte d'entreprendre, à travers l'acquisition de compétences, la constitution de réseaux, l'évolution des représentations identitaires» (Charles Pauvers, Schieb-Bienfait, 2005, p. 16). L'exploitation des données recueillies nous a conduits à formuler plus largement notre problématique: entrer en coopérative modifie-t-il le rapport au travail?

La première partie de l'article mobilisera la littérature sur la CAE, les profils génériques d'entrepreneurs, leurs compétences et leur identité professionnelle. Dans la seconde partie, nous présenterons une enquête exploratoire dans une de ces coopératives, qui infirme certains aspects de la revue de littérature.

\section{La coopérative d'activités et d'emploi (CAE) et ses entrepreneurs-salariés}

\section{Les particularités de la CAE}

Dernière nées dans la catégorie des Scop en France, les CAE sont fédérées par deux réseaux: Coopérer pour entreprendre (le principal, avec plus de soixante-dix CAE) et Copea (une trentaine). Elles accompagnent environ 6500 entrepreneurs-salariés (E-S).

\section{D'ABORD POUR LES INDIVIDUS}

Le premier exemple de ces nouvelles formes d'organisations coopératives est apparu il y a vingt ans: "Née en novembre 1995, la Scop Cap Services s'est donné pour métier d'accompagner celles ou ceux qui, face à la dégradation de l'emploi, ont décidé de créer leur propre activité» (Giacometti, 2001, p. 154). Son fonctionnement évolue avec le temps: "Cap Services ne devient une CAE qu'au fil du temps, à mesure que ses concepteurs ajustent leurs réponses aux problèmes des porteurs de projets» (Giacometti, 2005). "Elle est d'abord une couveuse - qui est elle-même l'aboutissement d'une réflexion menée dans un cadre associatif-, puis une coopérative d'activités" (Draperi, 2014, p. 218). La CAE s'adresse surtout à des entrepreneurs qui sont demandeurs d'emploi. 
"L'objectif premier de la CAE est d'offrir à des porteurs de projet souvent issus du chômage une alternative à l'immatriculation d'une entreprise individuelle » (Delvoldé, Veyer, 2011, p. 79). La CAE va en effet proposer en quelque sorte un parcours d'entrepreneur: celui-ci va pouvoir expérimenter son projet de création en tant qu'entrepreneur accompagné, puis devenir entrepreneursalarié, avant de passer éventuellement entrepreneur associé (Avise, 2009), voire de quitter la CAE pour créer une structure indépendante.

La CAE innove ainsi juridiquement: "Le mouvement des coopératives d'emploi souhaite la reconnaissance d'un statut particulier (entrepreneurs-salariés ou salariés-entrepreneurs) pour les salariés non coopérateurs tenant compte de leur double situation: salariés de la coopérative et véritables travailleurs indépendants» (Laurent, Mouriaux, Mundler, 2006, p. 41).

Cette innovation pose évidemment la question de la subordination: "La nature de la subordination dans une CAE questionne à chaque fois en ce qu'elle n'épouse pas un schéma classique du salariat» (Delvoldé, Veyer, ibid, p. 84). "Les CAE sont en effet construites sur le refus de la subordination» (Bodet, de Grenier, Lamarche, 2013, p. 37). Ce statut original relève sans doute de la parasubordination, à mi-chemin entre salariat et entrepreneuriat (Hernandez, 2007).

\section{LA NATURE DE LA STRUCTURE}

Parmi les structures d'accompagnement entrepreneurial, les CAE se différencient en particulier des sociétés de portage. "Il s'agit bien d'un portage mais sans finalité lucrative et qui s'inscrit dans le cadre d'une démarche économique collective. [...] Ces coopératives se distinguent des autres formes de portage d'abord en tant que coopérative (entreprise commune, société de personnes, dont tous les participants ont des droits égaux indépendamment du montant de leur apport en capital)»(Mouriaux, 2006, p. 20).

De plus, les individus qui travaillent dansles CAE sont attachés à ces différences, en particulier les dirigeants: "Dans le cas des gérants de coopératives d'activités et d'emploi, le statut juridique de leur entreprise (Scop) est érigé en symbole de ce refus [de l'entreprise classique]. Les sociétés de portage salarial, pourtant situées sur le même créneau d'activité mais qui sont des entreprises classiques, constituent pour les gérants de coopérative des figures repoussoirs auxquelles il convient pour eux de ne pas être assimilés» (Darbus, Lazuech, 2010, p. 5).

\section{LA DIMENSION COLLECTIVE}

L'aspect collectif se retrouve naturellement dans les CAE. "L'ambition première de Cap Services, lorsqu'elle fut créée en 1995, était de sécuriser les premiers pas de porteurs de projets ayant l'intention de créer leur entreprise individuelle. [...] Les CAE de deuxième génération ne visent plus à sécuriser la création d'entreprises individuelles, mais bien à construire une alternative à celles-ci, via un projet d'entrepreneuriat collectif » (Sangiorgio, Veyer, 2009, p. 55-56).

Ainsi, les CAE assurent à la fois un accompagnement individualisé et collectif des porteurs de projets et un statut de salarié. Elles constituent un cadre économique, juridique et social à même d'accueillir, d'accompagner et d'héberger des projets d'activités économiques de tous types. Elles poursuivent un objectif de développement collectif et solidaire d'activités, en 
s'inscrivant dans une logique d'insertion par l'économique. Cet accompagnement collectif pourrait d'ailleurs être assimilé à une forme de néocompagnonnage (Noël, Stervinou, 2008).

\section{Les profils d'entrepreneurs}

\section{LES DEMANDEURS D'EMPLOI}

De nombreux auteurs indiquent que beaucoup d'entrepreneurs sont des demandeurs d'emploi qui, ne retrouvant pas d'activité au travers du « sacrosaint CDI à temps plein mono-employeur ", cherchent à construire la leur. Le chômage pousse en effet de plus en plus de demandeurs d'emploi à créer leur propre entreprise et ainsi leur emploi. "Près d'une entreprise sur deux est créée par une personne au chômage ou à la recherche d'un emploi " (Charles Pauvers, Schieb-Bienfait, 2010, p. 107). La logique d'action de la réinsertion professionnelle concerne des acteurs qui vivent des situations de rupture professionnelle: très souvent demandeurs d'emploi en difficulté pour retrouver un emploi salarié, ils s'appuient sur leur expérience, leurs espaces d'opportunités et l'ensemble de leurs ressources disponibles pour se réinsérer professionnellement et socialement, en utilisant la voie entrepreneuriale (Fayolle, 2003). On notera au passage que l'un des traits de caractère prêtés aux entrepreneurs, à savoir une forte confiance en eux, ou du moins en leur bonne étoile (Julien, Marchesnay, 1996), est sans doute moins présent dans ce cas.

Les entrepreneurs de CAE sont souvent des personnes en situation de chômage ou vivant des minima sociaux (Noël, Stervinou, 2008): " $80 \%$ des candidats à l'entrepreneuriat collectiffont l'expérience de périodes plus ou moins longues et répétées de chômage » (Darbus, 2006, p. 30).

\section{LES SALARIÉS}

Les autres entrepreneurs sont encore salariés et hésitent à quitter leur poste pour se lancer de manière indépendante. Leurs motivations peuvent naître et s'exprimer alors qu'ils sont encore chez leur employeur, au sens où "chaque membre de l'organisation est une entreprise à part entière » (Hernandez, 2001, p. 235). On peut alors parler d'intrapreneur (Carrier, 2000). Nous ne développerons cependant pas cet aspect, dans la mesure où aucun des interviewés de notre enquête n'était salarié à l'entrée dans la CAE.

\section{Compétences et identité professionnelle des entrepreneurs-salariés}

"De par ses fondements, l'économie sociale et solidaire constitue un projet très ambitieux et prometteur pour les ressources humaines " (Everaere, 2011, p. 17). Elle n'implique pas pour autant des supports originaux. Ainsi, dans les Scop qui nous intéressent plus particulièrement, les outils de gestion de ressources humaines (GRH) ne sont pas fondamentalement différents de ceux de l'entreprise classique (Mathé, Rivet, 2003).

De même, le vocabulaire utilisé dans celle-ci peut générer un rejet dans celleslà: par exemple, "le concept de GRH est souvent mal perçu par les dirigeants d'économie sociale parce qu'il est associé dans leur esprit à une conception 
capitaliste des travailleurs comme constituant une "ressource" à exploiter au même titre que les autres ressources (financières, matérielles, énergétiques, etc.) de l'entreprise» (Davister, 2006, p. 10).

Malgré tout, comme dans tout collectif de travail, les dirigeants de l'ESS ont à traiter "les problématiques de management, autour de la question clé des compétences» (Everaere, 2011, p. 21).

\section{LES COMPÉTENCES}

En France, la gestion des compétences a fait l'objet d'une littérature foisonnante, particulièrement depuis le début des années 90. Si des dizaines de définitions ont été énoncées, "ily a un relatif accord sur ce qu'est une compétence individuelle: une combinaison de ressources - liées à l'expérience ou à la formation de la personne, mais aussi à la situation de travail dans laquelle elle se trouve - qui rend une personne au travail "capable de...", dans un contexte précis» (Defélix, Klarsfeld, Oiry, 2006, p. 2).

Plus particulièrement pour les CAE, "les conditions d'entrée sont simples: disposer d'un savoir-faire dont on souhaiterait vivre de manière autonome; être prêt à mettre en œuvre ce savoir-faire sans attendre et sans que cela requiert de gros investissements financiers; avoir envie de mettre en œuvre ce savoir-faire dans un cadre collectif hétérodoxe dont on a bien compris les règles et l'esprit " (Sangiorgio, Veyer, 2006, p. 90).

Cette volonté affichée se heurte cependant à la réalité du profil des individus: "Bien que relativement qualifiés (près de $67 \%$ ont au moins le bac, contre un peu plus de $30 \%$ pour l'ensemble de la population), [nombre de candidats] sont cependant porteurs de titres scolaires peu rentables et dévalués »(Darbus, 2006, p. 28). Seuls «certains sont titulaires de compétences et de qualifications qui les protègent du chômage dans la mesure où, dans leur branche, le travail ne manque pas" (p. 31).

Par ailleurs, il semble généralement souhaitable d'articuler la gestion des compétences individuelles et des compétences collectives (Le Boterf, 2001) - celles-ci sont liées de manière non univoque à la performance (Bataille, 2001). Pour définir ces dernières, Krohmer et Retour (2006, p. 152) distinguent " quatre attributs essentiels constitutifs de la compétence collective-le référentiel commun, le langage partagé, la mémoire collective et, enfin, l'engagement subjectif».

Dans le contexte particulier des CAE, l'apport des compétences devrait alimenter les compétences collectives: "Les compétences diverses des coopérateurs salariés associés font la force du réseau et du collectif. Elles peuvent être sollicitées pour des besoins divers » (Allard et al., 2013, p. 77). D’ailleurs, la proposition de ces CAE est aujourd'hui de "partager une entreprise entre entrepreneurs pour mutualiser les compétences et se donner collectivement des droits, de la sécurité et des opportunités » (Sangiorgio, Veyer, 2006, p. 92). Nous pouvons mentionner ici que, indépendamment des spécificités du contexte, «la création d'un collectif repose sur les multiples interactions entre les membres qui le composent mais, simultanément, les compétences collectives ont des impacts sur le développement des membres du collectif» (Defélix, Picq, Retour, 2009, p. 214). Pour faire le lien avec l'identité professionnelle, que nous souhaitons également approfondir dans le cas des CAE, citons Chédotel et Pujol (2009) qui, à propos des Scop, ont cherché à «montrer que les processus d'identification 
sociale jouent un rôle déterminant pour passer du niveau individuel au niveau collectifde compétence» (p. 79), sachant que "les Scop revendiquent une identité forte et duale, c'est-à-dire à la fois fondée sur des valeurs entrepreneuriales (compétitivité) et des valeurs d'économie sociale (solidarité, démocratie, primautéde la personne)» (p. 84).

\section{L'IDENTITÉ PROFESSIONNELLE}

Pour Sainsaulieu (1985, p. IX), " [1'identité au travail], façon dont les différents groupes au travail s'identifient aux pairs, aux chefs, aux autres groupes, [...] est fondée sur des représentations collectives distinctes, construisant des acteurs du système social d'entreprise».

Nous lui préférerons la définition de Dubar (2000, p. 109), selon lequel "l'identité n'est autre que le résultat à la fois stable et provisoire, individuel et collectif, subjectifet objectif, biographique et structurel, des divers processus de socialisation qui, conjointement, construisent les individus et définissent les institutions ».

En CAE, l'identité professionnelle semble liée au statut d'E-S: «Changeant de statut pour être entrepreneur-salarié, certains recourent à de nouvelles identités plus rentables » (Darbus, 2006, p. 32). Ce statut rend malléable leur identité: "La volonté de reconnaissance de leur singularité est particulièrement exprimée par les membres des coopératives d'emploi et d'activités. [...] Ils revendiquent la reconnaissance d'un statut de "salariés-entrepreneurs" qui entérinerait leur appartenance à une communauté de travailleurs à la fois autonomes et solidaires. L'identité n'est pas figée " (Laurent, Mouriaux, Mundler, 2006, p. 47).

Plus généralement, les phénomènes (aléas des marchés, reconfiguration des alliances avec des partenaires, déplacement de l'investissement, qui glisse des activités productives vers des activités de négociation de prestations et de transformation du savoir) à l'origine de l'émergence des nouvelles formes d'organisation et d'emploi (comme la CAE) génèrent en effet un flou, voire une rupture identitaire, dont souffrent les salariés (Freiche, Le Boulaire, 2000). Ceux-ci tendent alors à perdre leur identité d'entreprise (Dubar, 2000). Cette dernière est l'une des quatre formes identitaires de la typologie établie par l'auteur. Celles-ci peuvent s'interpréter à partir des modes d'articulation entre transaction objective et transaction subjective, comme des résultats de compromis «intérieurs » entre identité héritée et identité visée, mais aussi de négociations "extérieures » entre identité attribuée par autrui et identité incorporée par soi.

Les identités construites sur le mode de la continuité impliquent un espace de réalisation, de type organisationnel (entreprise) ou professionnel (métier). Celles qui sont construites sur le mode de la rupture impliquent une dualité entre l'espace de production de son identité passée et l'espace de construction de son identité future.

En cas de reconnaissance, l'identité visée est légitimée par une institution (entreprise, organisme de formation). En cas de non-reconnaissance, l'avenir de l'institution ne coïncide pas avec l'avenir de l'individu.

Nous mobiliserons la grille de lecture présentée (tableau 1, en page suivante) pour positionner certains interviewés de notre enquête de terrain. 


\section{Tableau 1}

Les quatre processus identitaires typiques selon C. Dubar

\begin{tabular}{|l|l|l|l|}
\hline \multirow{2}{*}{$\begin{array}{l}\text { Identité } \\
\text { pour soi }\end{array}$} & \multirow{2}{|l|}{$\begin{array}{l}\text { Identité } \\
\text { pour autrui }\end{array}$} & Transaction objective \\
\cline { 3 - 4 } & Reconnaissance & Non-reconnaissance \\
\hline $\begin{array}{l}\text { Transaction } \\
\text { subjective }\end{array}$ & Continuité & $\begin{array}{l}\text { Promotion (interne) } \\
\text { Identitéd'entreprise }\end{array}$ & $\begin{array}{l}\text { Blocage (interne) } \\
\text { Identitéde métier }\end{array}$ \\
\cline { 2 - 4 } & Rupture & $\begin{array}{l}\text { Conversion (externe) } \\
\text { Identitéde réseau }\end{array}$ & $\begin{array}{l}\text { Exclusion (externe) } \\
\text { Identitéde hors-travail }\end{array}$ \\
\hline
\end{tabular}

\section{Une recherche exploratoire au-delà des compétences et de l'identité}

Dans un article de 2007, Draperi oppose aux deux traditions, universitaire (classique, fonctionnaliste) et critique, la tradition de pensée de l'économie sociale qui promeut la recherche-action, et dont il dresse une liste d'auteurs spécifiques. Nous pourrions y ajouter le nom d'Henri Fayol (Peaucelle, 2000), bien que ce dernier soit abondamment cité par les tenants de la tradition fonctionnaliste. Surtout, dans ce même article, Draperi indique que "la mise en œuvre par la (les) même(s) personne(s) d'une recherche-action articulant les deux métiers, exercés professionnellement ou non, de chercheur et d'acteur définit l'éducation et l'émancipation spécifiques à l'économie sociale» (p. 73). Nous pensons nous être inscrits dans cette lignée, mais de façon diachronique: juste après avoir travaillé dans une CAE pendant dix-huit mois, nous avons participé à un colloque sur le management des Scop (dont font donc partie les CAE) et publié un chapitre d'ouvrage (Vilette, 2009). A cet effet, nous avons alors mené une enquête auprès d'E-S de la CAE dans laquelle nous avions opéré, enquête modeste tant par le nombre d'interviewés que par l'incomplétude du guide d'entretien (dont les compétences et l'identité étaient les principaux items). Nous n'avions alors identifié qu'une seule publication sur les CAE. Notons néanmoins que, sur les trois autres antérieures à notre enquête (parmi la douzaine que nous mentionnons aujourd'hui), deux ne parlent que de Cap Services, la première créée.

C’est donc au regard d'une bibliographie mobilisée considérablement étoffée (et qui dépasse le cadre des CAE) que nous avons réexploité les données recueillies initialement et formulé notre problématique. Pour ce faire, l'une des difficultés tient aux éléments (non verbaux par exemple) ne figurant pas sur les notes d'entretien et qui sont naturellement moins présents à l'esprit en raison du temps écoulé.

\section{Méthodologie}

Le terrain d'étude est la CAE dans laquelle nous avons travaillé. Implantée en Haute-Savoie et adhérente du réseau Copea, elle a connu une forte croissance, puisque, créée par six cofondateurs, elle comptait cinquante et un E-S au bout de cinq ans, malgré quelques départs. 
C'est à ce moment-là que nous avons mené notre enquête, en ayant recours à une méthodologie qualitative de nature exploratoire. "Une recherche ne devrait être qualifiée d'exploratoire que si elle l'est réellement, c'est-à-dire si, de près ou de loin, le sujet n'a jamais ététraité» (Igalens, Roussel, 1998, p. 73). Or, comme nous l'avons déjà indiqué, assez peu de travaux ont été publiés sur la CAE aujourd'hui, et moins encore lors de l'enquête.

La composition de l'échantillon s'explique par la recherche d'une diversité d'angles de vue. Elle passe par la prise en compte de différences en termes d'âge (de 40-45 ans à 50-55 ans) et d'ancienneté (de quinze mois à cinq ans), de spécialité (du traitement de l'image au droit) et de niveau d'études (de $\mathrm{bac}+1$ à bac +5 de formation initiale), ainsi que de type d'activité. Précisons que, si la plupart des membres de cette CAE étaient qualifiés et expérimentés, cette diversité est néanmoins limitée par le nombre restreint d’interviewés. D'autre part, pour des raisons de proximité et de réseau social lié à notre propre pratique, notre échantillon est constitué d'E-S de la CAE dans laquelle nous avons exercé. "Une recherche exploratoire comporte souvent une collecte de données effectuée sur un échantillon de convenance» (Igalens, Roussel, 1998, p. 73). Cela a sans doute facilité la programmation des entretiens dans des délais courts (liés alors au colloque sur les Scop). Cependant, la connaissance mutuelle est source de biais potentiels, sur lesquels nous reviendrons en conclusion.

Notons par ailleurs que nous avons partiellement utilisé la méthode «boule de neige »: par exemple, une fois l'un des entrepreneurs identifié et interviewé, nous lui avons demandé de nous recommander à un autre entrepreneur, que nous ne connaissions pas, puisqu'il n'était pas engagé dans la CAE lorsque nous y avons œuvré.

Nous avons mené des entretiens semi-directifs centrés (Romelaer, 2005) d'une durée comprise entre quarante-cinq minutes (le seul par téléphone) et une heure quarante-cinq. Parmi les thèmes du guide initial de l'interviewer figuraient certains de ceux qui étaient proposés en vue du colloque initial, en particulier les compétences et l'identité professionnelle.

Le matériel recueilli a donné lieu à une analyse de contenu (contenu du discours pour un entretien semi-directif) « semi-formatée », puisqu'une partie des thèmes a été fixée avant l'entretien, qui laissait cependant la possibilité de découvrir de nouveaux thèmes au cours de l'analyse de contenu. Celle-ci vise à réduire les informations afin de les catégoriser et de les mettre en relation avant d'aboutir à une description ou à une explication (Wacheux, 1996).

\section{Résultats et discussion}

\section{LE VÉCU DANS LA CAE RÉORIENTE LE PROCESSUS IDENTITAIRE DES INTERVIEWÉS}

Tous les interviewés reconnaissent que leur identité professionnelle a évolué avec leur parcours dans la CAE (malgré la diversité de sa durée), mais pas pour les mêmes raisons: deux parlent d'un changement de métier (d'opérationnel à conseil pour l'un, à formateur pour l'autre), le troisième estime que le parcours « a pu [1]'aider à y voir plus clair», les deux autres soulignent que la confrontation au marché a permis d'éclaircir leurs compétences. Illustrons par l'exemple d'Isabelle (encadré1, en page suivante). 
Si nous cherchons à utiliser la grille de Dubar pour nos interviewés, nous pouvons considérer que tous étaient en rupture quand ils ont intégré la CAE. Annie (encadré 2, en page suivante), quant à elle, est sans doute dans un processus identitaire d'entreprise (en devenant permanente à mi-temps c'est-à-dire que la CAE lui fournit une activité assurant un salaire à $50 \%$ d'un équivalent temps plein (ETP) - pour accompagner les nouveaux arrivants). Isabelle (encadré1) semble glisser vers une identité de hors-travail, eu égard à ses difficultés à générer du chiffre d'affaires et à ses activités bénévoles dans des domaines culturels connexes. Enfin, Patrice (encadré3, en page 79) tendrait vers une identité de réseau, au titre de la formation suivie pour préparer un diplôme professionnel.

Si nous nous sommes initialement plutôt intéressés à l'identité individuelle, nous avons néanmoins rappelé l'identité forte et duale des Scop (Chédotel, Pujol, 2009).

Or, dans la CAE étudiée, alors que les E-S se présentent au titre de la CAE dans leurs démarches commerciales, ils ne s’identifient pas (ou peu) à celle-ci, de par leur relative autonomie de fonctionnement: "Ce sont mes clients, pas ceux de la coopérative. »

D’ailleurs, leur représentation de l'identité de celle-ci est très hétérogène: «il y a encore du boulot pour la définir », "diversitéet rigueur», "en croissant, elle devient une entreprise comme une autre", "une pépinière de projets d'entrepreneuriat", "la cohabitation de deux systèmes: des entrepreneurs-salariés qui ont roulé leur bosse et un collège d'apprentis ».

Cette difficulté vis-à-vis de l'identité collective va également se retrouver au niveau des compétences.

\section{LA DIMENSION COOPÉRATIVE NE PERMET PAS LA CONSTRUCTION DE COMPÉTENCES COLLECTIVES À PARTIR DES COMPÉTENCES INDIVIDUELLES}

Interrogés sur leurs compétences, tous les E-S parlent spontanément de celles en lien direct avec le(s) métier(s) qu'ils exercent - ou ont exercé(s) -, à l'exclusion de celles que la littérature prête au créateur d'entreprise, qu'elles soient à dominante individuelle, sociale et même cognitive (Charles Pauvers et alii, 2004). Faut-il alors émettre l'hypothèse que ces E-S sont encore plutôt salariés qu'entrepreneurs? Tous soulignent pourtant la particularité de devoir «aller chercher le travail» (mais à regret, pour certains).

\section{Encadré 1}

Isabelle a la quarantaine, et quatre ans et demi d'ancienneté dans la CAE. Titulaire d'une maîtrise en sciences de l'information, elle est successivement enseignante de latin, chargée de communication en grande entreprise, auteure d'un livre. Elle a passé deux ans à l'étranger. Elle essaie de développer une activité de journalisme d'entreprise. "J'ai évolué, ça ne fait pas l'ombre d'un doute. J'ai éclairci mes compétences, ce qui est une obligation pour la démarche commerciale, carj'avais essuyé tellement d'échecs. En fait, je me suis construit une identité professionnelle à partir d'un puzzle.» 
Par ailleurs, tous évoquent la richesse de la diversité des E-S et l'intérêt que présente la CAE en termes d'échanges, d'ouverture, de découverte de métiers, de réseaux. Cependant, ils constatent également la difficulté à associer des compétences trop éloignées. Mais même dans les axes spécialisés (regroupant des E-S travaillant respectivement dans la communication et l'industrie), les collaborations concrètes et les contributions communes restent trop rares. Ainsi, si la croissance de la CAE est significative, la dimension collective reste plus difficile à appréhender que dans une Scop classique, de par la diversité des projets individuels. Leur addition ne semble pas faire un projet collectif. Cette difficulté se retrouve cependant dans la littérature postérieure à l'enquête. Ainsi, "certaines CAE, nées sous le signe de la première génération, rencontrent de nombreuses difficultés à migrer vers le modèle d'entreprise partagée » (Ribeiro, Vignaud, 2010, p. 15).

\section{LA PRIMAUTÉ SOCIALE N'EXCLUT PAS L'ASPECT ÉCONOMIQUE}

Comme indiqué précédemment, pour la plupart des publications surl'économie sociale, "c'est toute l'idéologie même de l'ESS qui fait de l'amélioration du sort de l'humain le but même des activités économiques, cecien rupture avec le système capitalisteclassique»(Everaere, 2011, p. 18). Or, plusieurs interviewés indiquent un positionnement moins marqué de leur CAE. C'est le cas d'Annie (encadré2). Cette différence avec la littérature dominante se retrouve néanmoins chez certains auteurs: ainsi, un glissement "conduit les structures de l'économie sociale, en particulier celles qui se trouvent directement confrontées aux règles du marché, à se rapprocher du point de vue des pratiques managériales des entreprises classiques. Ce glissement est particulièrement visible du côté des coopératives d'activités et d'emploi » (Darbus, Lazuech, 2010, p. 17).

\section{LA COPROPRIÉTÉ DE LA COOPÉRATIVE ET LA PRISE DE DÉCISIONS ASSOCIÉE NE SONT PAS TOUJOURS VALORISÉES}

Comme mentionné initialement, la participation au capital donne au coopérateur un pouvoir de décision. Celui-ci est censé l'impliquer davantage dans la coopérative. "La participation aux décisions est souvent avancée comme une source importante de motivation et de coopération entre les membres.

\section{Encadré 2}

Annie approche la cinquantaine. Elle est entrée dans la CAE il y a quinze mois. Ingénieur informatique de l'Institut supérieur des métiers, elle a d'abord été manager d'équipe support chez un grand constructeur, avant d'évoluer vers la fonction " ressources humaines » (RH). Elle développe aujourd'hui une activité d'accompagnement et de formation en management et en communication.

"La dimension économique est moins présente. La rentabilité n'est pas dans le discours coopératif. Il y a une certaine ambiguïté entre la rentabilité et le social dans notre coopérative. Elle a un positionnement très particulier, avec plus le souci de rentabilité que dans les autres, mais sans déperdition de la dimension humaine. » 
L’idée principale est que les salariés s'investiront d'autant plus dans leur travail qu'ils se sentiront en mesure de peser sur les décisions qui les concernent et concernent leur entreprise» (Fauvy, 2009, p. 145).

Cette caractéristique se retrouve assez logiquement dans les CAE. «Le fait que tout salarié peut devenir associé fait tendre à choisir non plus seulement sur des critères "techniques", mais aussi sur une appétence à la copropriété de l'outil de production et à la finalité sociale de l'entreprise (citation de CS, coopérative d'activités et d'emploi)»(Everaere, 2011, p. 26).

Pourtant, dans la CAE étudiée, l'importance accordée au sociétariat et l'engagement qu'il induit ne sont pas soulignés par ceux qui vont y parvenir.

\section{TRAVAILLER DANS UNE COOPÉRATIVE N'EST PAS UN CHOIX DÉLIBÉRÉ POUR LES INTERVIEWÉS}

Comme la méthodologie en indiquait la possibilité, ce premier thème ne figurait pas dans le guide d'entretien, mais a émergé dans l'analyse de contenu. Nous ne l'avons pas identifié non plus dans la littérature.

Tous les interviewés avaient bien dans l'idée de créer ou de poursuivre une activité autonome, en rupture avec le salariat classique. Cependant, aucun n’a volontairement fait le choix de la coopérative, à l'issue d'une réflexion au sujet du statut adopté.

Ainsi, le cofondateur songeait plutôt à recourir à une société de portage (une autre interviewée l'a d'ailleurs expérimenté). Mais il s'est rallié à la proposition d'un autre cofondateur, qui connaissait le cadre original de la CAE au travers d'une activité précédente (et qui deviendra ensuite le directeur). Quant aux autres, ce sont plutôt les circonstances qui ont joué: l'un a lu un article sur la CAE dans la presse économique locale, un autre est venu sur recommandation d'un entrepreneur-salarié avec qui il était en relation, les deux derniers ont été mis en relation par l'intermédiaire de l'association locale Compétences en temps partagé (CTP), hébergée par la CAE. D’ailleurs, tous les interviewés connaissent le travail à temps partagé et quatre considèrent l'exercer au sein de la CAE (Vilette, 2009). Paradoxalement, le seul à avoir une perception différente est le cofondateur, alors qu'il était adhérent

\section{Encadré 3}

Patrice a la quarantaine, et trois ans et demi d'ancienneté dans la CAE. Après une mâ̂trise de droit, il est durant deux ans vacataire dans l'Education nationale. Il se lance ensuite dans l'administration de biens. Les douze ans d'expérience dans ce domaine s'interrompent brutalement. Il suit alors une formation de formateurs, pour dispenser de la formation en droit immobilier. Insatisfait par le portage lors des deux premières années d'activité, il entre à la CAE.

"J'ai obtenu des aides variées: du conseil, de l'assistance, de la présence, de l'amitié. Ça crée des liens entre les individus. Je me suis senti mis à l'aise, j'ai été soutenu. Et aujourd'hui, avec la taille, la structuration, la professionnalisation, ça prend une dimension fonctionnelle. » (Création d'un poste d'accompagnement à mi-temps, sur lequel est d'ailleurs recrutée Annie.) 
de cette association au moment de la création de la CAE, comme les cinq autres cofondateurs (le futur directeur ayant même été cofondateur, puis président de CTP).

Tentons d'analyser cette absence « d'appétence " pour la coopérative. Tout d'abord, si le travail à temps partagé fait partie pour certains, comme indiqué précédemment, de leurs trajectoires de vie, celles-ci ne leur ont pas donné l'occasion de connaître le milieu coopératif.

Ensuite, en termes d'attentes vis-à-vis de la CAE, tous évoquent l'aide administrative (factures, salaires, tenue des comptes), que l'on peut obtenir dans d'autres types de structure. Ils mentionnent également l'accompagnement (souvent par différence avec le portage), plus caractéristique du contexte coopératif, à l'image de Patrice (encadré 3 , en page précédente).

En revanche, seuls deux d'entre eux citent d'abord la liberté ("On n'est pas managés»), alors que celle-ci est souvent évoquée parmi les motivations prioritaires du créateur d'entreprise. D’ailleurs, ces deux E-S sont sans doute également ceux qui fonctionnent comme tel. Les autres semblent plutôt peiner à développer leur activité pour s'éloigner du statut de demandeur d'emploi.

\section{Conclusion}

La confrontation de la littérature aux données de terrain nous indique qu'entrer en coopérative modifie en effet le rapport au travail, mais de manière contrastée.

Ainsi, le processus identitaire en est effectivement affecté. Toutefois, l'image positive affichée sur le plan collectif - «chaque CAE, qui naît comme structure d'appui à la création d'activité et grandit comme entreprise à mesure de son appropriation par ses entrepreneurs-salariés [...], qui est conçue pour accompagner des démarches entrepreneuriales individuelles et qui nourrit sa croissance des pratiques entrepreneuriales collectives qu'elle génère " (Sangiorgio, Veyer, 2006, p. 92) - n'est pas reflétée par les interviewés: les compétences collectives ne parviennent pas à se construire, ce qui peut en partie s'expliquer par le fait que «la CAE n'est plus tout à fait une coopérative de production. Elle est une coopérative d'éducation coopérative» (Draperi, 2014, p. 221).

De même, la priorité du social qui peut avoir guidé l'E-S dans la coopérative n'exonère cependant pas d'une certaine résurgence des impératifs économiques dans le rapport au travail.

En outre, l'engagement dans le travail lié à la détention partielle du capital n'est pas toujours au rendez-vous.

Quant au choix non délibéré de travailler dans une coopérative (que nous n'avions pas repéré dans la littérature), il ne vient pas appuyer l'idée que "pour ces nouveaux dirigeants [de l'économie sociale], la création d'entreprise "autre" est largement liée au fait qu'ils ne se reconnaissaient plus dans les objectifs et les méthodes managériales employées au sein de la fonction publique ou de l'entreprise classique» (Darbus, Lazuech, 2010, p. 16).

Nous pouvons émettre deux hypothèses explicatives à ces résultats contrastés: - l'appartenance au réseau Copea, plutôt que Coopérer pour entreprendre; la fondatrice de la première CAE indique ainsi que les «coopératives d'emploiet 
d'activitédu réseau Copea [...], n’hésitent pas à parler de "portage salarial sous forme coopérative" [...] faisant fi des conditions nécessaires à une démarche collective d'entreprise, [ou] font obligation, dès l'entrée, d'adhésion au sociétariat» (Bost, 2011, p. 142-143);

- même dans les CAE, "bien que s'adressant à une coopérative, les porteurs de projets ne s'inscrivent pas dans une démarche coopérative, ne serait-ce que parce qu'ils l'ignorent fréquemment» (Draperi, 2011, p. 173).

En outre, deux limites de la recherche sont également à mentionner:

- le mode d'accès aux interviewés (réseau personnel) est source de biais potentiels, comme indiqué précédemment. Bien que nous n'ayons perçu de gêne à aucun moment, la connaissance mutuelle peut en effet avoir inhibé quelque peu l'expression. Cependant, nous pensons qu'elle l'a plutôt facilitée, certaines informations sensibles nous ayant été livrées (mais non reproduites dans cet article, car elles n'étaient pas en lien direct avec notre problématique actuelle);

- nous n'avons pas distingué les interviewés selon leur ancienneté (donc leur avancement dans l'accompagnement et leur situation de coopérateur), car elle ne nous a pas semblé différencier leurs réponses. Cet aspect aurait cependant pu faire l'objet de davantage de vigilance.

Au-delà de pallier ces limites, cette enquête offre d'autres perspectives de recherche. D’une part, afin de dépasser ce regard à un instant donné (ce qui semble réducteur pour bien comprendre un processus d'accompagnement - et donc son influence sur les compétences et l'identité des E-S), il semble nécessaire de revisiter le terrain étudié, dans une optique longitudinale. Cela permettrait également d'enrichir le guide d'entretien initial autour des aspects sur lesquels nous nous sommes centrés.

D'autre part, outre la distinction selon l'ancienneté, nous pourrions chercher à obtenir une triangulation des données, en réalisant des entretiens auprès des permanents (salariés, dont le directeur) de la coopérative, mais aussi d'anciens E-S qui ont quitté la CAE (d'ailleurs, aucun interviewé initial ne figure sur le site actuel de la CAE).

Enfin, nous nous sommes volontairement focalisés sur des individus dont le niveau de qualification est plutôt élevé - que nous retrouvons d'ailleurs dans la CAE leader qu'est Coopaname (Bodet, de Grenier, 2011) -, alors que la CAE concerne d'autres populations. La poursuite de nos échanges récents avec plusieurs CAE permettrait d'étudier lesdites populations. 


\section{BibliogRAPHIE}

\section{Allard F., Amans P., Bravo-Bouyssy K. et Loup}

S., 2013, «L'accompagnement entrepreneurial par les coopératives d'activités et d'emploi: des singularités à questionner », Management international, vol. $17, \mathrm{n}^{\circ} 1$, p. 72-85.

Avise, 2009, Coopératives d'activités et d'emploi: l'entreprise partagée, édition déléguée Bâton rouge-Polyprint édition.

Bataille F., 2001, " Compétence collective et performance", Revue de gestion des ressources humaines, ${ }^{\circ} 40$, p. 66-81.

Bodet C., de Grenier N., 2011, «Coopératives d'activités et d'emploi: des éléments de réponse de l'économie sociale au délitement du rapport salarial fordien ", Actes des XI ${ }^{\text {es }}$ rencontres $d u$ Riuess (Réseau universitaire de l'économie sociale et solidaire), Poitiers.

Bodet C., de Grenier N., Lamarche T., 2013, « La coopérative d'activités et d'emploi à la recherche d'un modèle productif ", Recma, n 329, p. 37-51.

Brasseur M., 2009, "Introduction ", in Brasseur M. (coord.), Entrepreneuriat et insertion, éd. Bruylant.

Bost E., 2011, Aux entreprenants associés: la coopérative d'activités et d'emploi, éd. Repas.

Carrier C., 2000, «L'intrapreneuriat: à la recherche de complices innovants et entreprenants ", in Verstraete T. (dir.), Histoire d'entreprendre, éd. Ems, p. 199-210.

Charles Pauvers B., Schieb-Bienfait N., Urbain C., 2004, « La compétence du créateur d'entreprise innovante: quelles interrogations? », Revue internationale PME, vol. 17 (1).

Charles Pauvers B., Schieb-Bienfait N., 2005, "L'entrepreneur-salarié, une forme innovante de maintien de l'identité professionnelle?", Actes du XVI congrès de l'Association francophone de gestion de ressources humaines (AGRH), Paris-Dauphine.

\section{Charles Pauvers B., Schieb-Bienfait N., 2010,}

«La compétence entrepreneuriale: la gestion des ressources humaines au service des démarches d'accompagnement, le cas des coopératives d'activités et d'emploi ", Gestion 2000, n 3, maijuin 2010, p. 107-120.
Charles Pauvers B., Schieb-Bienfait N., Urbain C., 2011, «Emergence entrepreneuriale et innovation sociale dans l'économie sociale et solidaire: acteurs, projets et logiques d'action ", in Flahaut E., Noguès H. et Schieb-Bienfait N., L'économie sociale et solidaire: nouvelles pratiques et dynamiques territoriales, Presses universitaires de Rennes, p. 53-77.

Chédotel F. , Pujol L., 2009, « De la compétence individuelle à la compétence collective: l'influence des processus d'identification sociale et le rôle du manager ", in Defélix C. Picq T. et Retour D. (coord.), Gestion des compétences: nouvelles relations, nouvelles dimensions, éd. Vuibert, p. 79-99.

Darbus F., 2006, « Reconversions professionnelles et statutaires: le cas des coopératives d'emploi et d'activités ", Regards sociologiques, $n^{\circ} 32$, p. 23-35.

Darbus F., Lazuech G., 2010, « Quelle identité pour les cadres de l'économie sociale?", Actes du $23^{e}$ colloque de l'Addes (Association d'aide au développement économique et social), Paris, p. 1-22.

Davister C., 2006, « La gestion des ressources humaines en économie sociale ", Les cahiers de la chaire Cera, $\mathrm{n}^{\circ} 1$, mai 2006.

Defélix C., Klarsfeld A., Oiry E., 2006, « Introduction ", in Defélix. C., Klarsfeld A. et Oiry E. (coord.), Nouveaux regards sur la gestion des compétences, éd. Vuibert, p. 1-9.

Defélix C., Picq T., Retour D., 2009, « Conclusion ", in Defélix C. Picq T. et Retour D. (coord.), Gestion des compétences: nouvelles relations, nouvelles dimensions, éd. Vuibert, p. 211-221.

Delvoldé N., Veyer S., 2011, « La quête du droit: approche de l'instauration d'une représentation du personnel dans une coopérative d'activités et d'emploi ", Recma, n 319, p. 78-96.

Draperi J.-F., 2007, «Fondements éthiques et posture épistémologique de la recherche en économie sociale ", Recma, n 303, p. 67-82.

Draperi J.-F., 2010, «L'entrepreneuriat social: du marché public au public marché », Recma, $\mathrm{n}^{\circ} 316$, p. 18-40. 
Draperi J.-F., 2011, « Postface », in Bost E., Aux entreprenants associés: la coopérative d'activités et d'emploi, éd. Repas, p. 173-175.

Draperi J.-F., 2014, Comprendre l'économie sociale:fondements et enjeux, éd. Dunod, 2e éd. Dubar C., 2000, La socialisation: construction des identités sociales et professionnelles, éd. Armand Colin.

Everaere C., 2011, « Valeurs et pratiques de GRH dans l'économie sociale », Revue française de gestion, $\mathrm{n}^{\circ} 217$, p. 15-33.

Fauvy S., 2009, "La mobilisation des personnels et les dispositifs de participation des ressources humaines dans les SCOP », in Laville J.-L. et Glémain P. (dir), L'économie sociale et solidaire aux prises avec la gestion, éd. Desclée de Brouwer, p. 139-164.

Fayolle A., 2003, Le métier de créateur d'entreprise, Editions d'Organisation.

Freiche J., Le Boulaire M., 2000, L'entreprise flexible et l'avenir du lien salarial, éd. L'Harmattan.

Giacometti D., 2001, «Coopérer pour entreprendre: de l'expérience de Cap Services à la création d'un réseau de promotion de l'entrepreneuriat collectif », Recma, n² 281, p. 154-161. Giacometti D. (coord.), 2005, Cap Services: mode d'emploi, éd. 10CAP.

Gianfaldoni P., Richez-Battesti N., 2005, «Réseaux d'accompagnement et de financement à la création des très petites entreprises: une analyse économique du surplus social ", in Amintas A., Gouzien A. et Perrot P. (dir), Les chantiers de l'économie sociale et solidaire, Presses universitaires de Rennes, p. 323-338.

Hernandez E.-M., 2001, L'entrepreneuriat: approche théorique, éd. L'Harmattan.

Hernandez E.-M., 2007, « Entre entrepreneuriat et salariat: la parasubordination ", in Fayolle A. (dir.), L'art d'entreprendre, Paris, coll. «Village mondial ", éd. Pearson, p. 79-85.

Igalens J., Roussel P., 1998, Méthodes de recherche en gestion des ressources humaines, éd. Economica.
Julien P.-A., Marchesnay M., 1996, L'entrepreneuriat, éd. Economica.

Krohmer C., Retour D., 2006, « La compétence collective, maillon clé de la gestion des compétences ", in Defélix. C., Klarsfeld A. et Oiry E. (coord.), Nouveaux regards sur la gestion des compétences, éd. Vuibert, p. 149-183.

Laurent C., Mouriaux M.-F., Mundler P., 2006, "Combinaison d'activités professionnelles et multifonctionnalités de l'agriculture: une résurgence de la dimension territoriale? », Document de travail, $\mathrm{n}^{\circ} 70$, Centre d'études de l'emploi, octobre 2006, p. 1-59.

Le Boterf G., 2001, Construire les compétences individuelles et collectives, Editions d'Organisation.

Mathé J.-C., Rivet A., 2003, "La Scop: une entreprise alternative?", Revue des sciences de gestion: direction et gestion, $\mathrm{n}^{\circ} 200$, p. 67-75.

Mouriaux M.-F., 2006, « Du fait au droit: diverses figures du temps partagé », Document de travail, $\mathrm{n}^{\circ} 77$, Centre d'études de l'emploi, décembre 2006.

Noël C., Stervinou S., 2008, « Les coopératives d'activités et d'emploi : un outil juridique au service d'un entrepreneuriat responsable", Revue Management \& Avenir, ${ }^{\circ} 20$, p. 65-86.

Peaucelle J.-L., 2000, "Henri Fayol et la recherche-action ", Gérer et comprendre, ${ }^{\circ} 62$, décembre 2000, p. 73-87.

Ribeiro E., Vignaud C., 2010, « Expérimentation et innovation sociale d'une coopérative d'activités et d'emploi ", Note de recherche, faculté de sciences économiques et de gestion, université Lumière-Lyon 2.

Romelaer P., 2005, "L'entretien de recherche ", in Roussel P. et Wacheux F. (dir.), Management des ressources humaines: méthodes de recherche en sciences humaines et sociales, éd. De Boeck, p. 101-137.

Sainsaulieu R., 1985, L'identité au travail, Presses de la fondation nationale des sciences politiques, $2^{e}$ éd.

Sangiorgio J., Veyer S., 2006, « L'entrepreneuriat collectif comme produit et projet d'entreprises 
épistémiques: le cas des coopératives d'activités et d'emploi », Revue de l'entrepreneuriat, vol. 5, $\mathrm{n}^{\circ} 2$, p. 89-102.

Sangiorgio J., Veyer S. 2009, « Les coopératives d'activités et d'emploi: un exemple de construction d'une innovation sociale », Projectique, $\mathrm{n}^{\circ} 1$, p. 51-61.
Vilette M.-A., 2009, «Entreprendre à temps partagé: la coopérative d'emplois et d'activités, pour accompagner l'insertion professionnelle", in Brasseur M. (dir.), Entrepreneuriat et insertion, éd. Bruylant, p. 229-244.

Wacheux F., 1996, Méthodes qualitatives et recherche en gestion, éd. Economica.

A RETROUVER DANS LES ARCHIVES DE LA « RECMA » (RECMA.ORG/ARTICLES)

Delvolvé N., Veyer S., « La quête du droit: approche de l'instauration d'une représentation du personnel dans une coopérative d'activités et d'emploi », Recma, n 319, 2011. Giacometti D., «Coopérer pour entreprendre: de l'expérience de Cap Services à la création d'un réseau de promotion de l'entrepreneuriat collectif ", Recma, n 281, 2001.

Darbus F., Hély M., « Travailler dans l’ESS: aspirations, représentations et dispositions. Etude auprès des adhérents de Ressources Solidaires », Recma, n³17, 2010. 